\title{
Western blot analysis of immune response to Legionella bozemanii antigens
}

\author{
I Boldur, H Kahana, R Kazak, B Cwikel, I Sarov
}

\begin{abstract}
The immune response to individual major antigens of Legionella bozemanii was studied in 67 sera from 26 inpatients with febrile disease using immunoblotting techniques. All the patients had fever of unknown origin and showed strong serological reactions to $L$ bozemanii that cross-reacted with Rickettsia typhi, as determined by a standard indirect microimmunofluorescence assay. Sera analysed by western blotting reacted with 12-14 molecular components of $L$ bozemanii with apparent molecular weights ranging from 14000 to 94000 daltons. These reactions compared well with a reference system using antisera of rabbits immunised with the same strain of Legionella. The three major cross-reactive components of $R$ typhi were polypeptide antigens of 94000,67000 and 43000 daltons.

It is concluded that western blotting can help in the differential diagnosis of patients with fever of unknown origin whose sera cross-react to $L$ bozemanii and $R$ typhi.
\end{abstract}

The usual serological assays for legionellosis are by immunofluorescence and agglutination. ${ }^{1-3}$ The specificity of these tests is high $(99 \%)$ when applied to patients with pneumonia, ${ }^{4}$ but for patients with other clinical symptoms, the specificity is unknown. Patients respond to Legionella infections by producing multiple antibodies which are serogroup specific, genus specific, and "nonspecific". These non-specific antibodies react with a variety of Gram negative bacteria but do not usually affect the specificity of the tests. $^{2}$

Serum samples from some patients with fever of undertermined aetiology are positive by indirect microimmunofluorescence assay (IFA) using either Legionella bozemanii serogroup 1 or Rickettsia typhi as antigen. Crossed immunoelectrophoresis shows the presence of a cross-reactive antigen to $L$ bozemanii. This antigen is heat stable and resistant to trypsin and is recognised by a reference rabbit anti- $L$ bozemanii serum as well as by a wide variety of human sera. ${ }^{5}$ The major $L$ pneumophila antigen recognised by human antisera in the IFA, and which determines the serogroup, is a lipopolysaccharidelike component probably found in association with the outer membrane proteins. ${ }^{67}$ Our study used the immunoblotting technique to characterise specific IgG and IgM antibodies to $L$ bozemanii in the sera of patients with fever which cross-react with $R$ typhi.

The main purpose of this study was to find a suitable procedure which would allow a diagnostic laboratory to provide an unequivocal serological evaluation of suspected cases of legionellosis.

\section{Methods}

Two or more serum samples were studied from 26 patients ( 11 women and 15 men, aged 30 to 58 years) from the department of internal medicine at the Assaf Harofeh Medical Center. All the patients had been admitted for high fever (over $39^{\circ} \mathrm{C}$ ) which had developed six to eight days previously. Repeated clinical examinations had failed to indicate any pathology. The patients complained of arthralgia, myalgia, malaise and headaches. Eight patients had some degree of respiratory disease, but an $x$-ray picture of the chest was normal. None of them had skin eruptions or other symptoms typical of any particular disease. All the patients were diagnosed as having fever of unknown origin, and the workup for such patients includes a urine sample, blood and sputum cultures (if available), and standard biochemical, haematological, and serological tests. The only findings were the significant titres for $L$ bozemanii (by immunofluorescence) and Proteus vulgaris OX19 in the Weil-Felix reaction. The sera were therefore checked for Rickettsiae (by immunofluorescence) and found to be positive for $R$ typhi.

Legionella cultures were from the Centers for Disease Control (CDC), Atlanta, Georgia. The serotypes of Proteus vulgaris (OX19, $\mathrm{OX} 2$, and $\mathrm{OXK}$ ) used for the standard WeilFelix test were from our strain collection and the test was performed as described in the CDC protocols. ${ }^{348}$ Sonicated cell antigens were prepared from a 72 hour growth on buffered charcoal yeast extract plates and McConkey agar for Legionella and Proteus strains, respectively. Cells were harvested in $3 \mathrm{ml}$ of water for each plate, and then washed three times by centrifugation and resuspension in water. The cells were finally resuspended in sterile distilled water, killed by ultraviolet irradiation, and sonicated for 10 one minute bursts under conditions which reduced turbidity to $20 \%$. $R$ typhi (Wilmington strain) was propagated in confluent Vero cell cultures. ${ }^{9}$ Infected Vero cells were harvested
Correspondence to: I Boldur

Accepted for publication 25 April 1991 


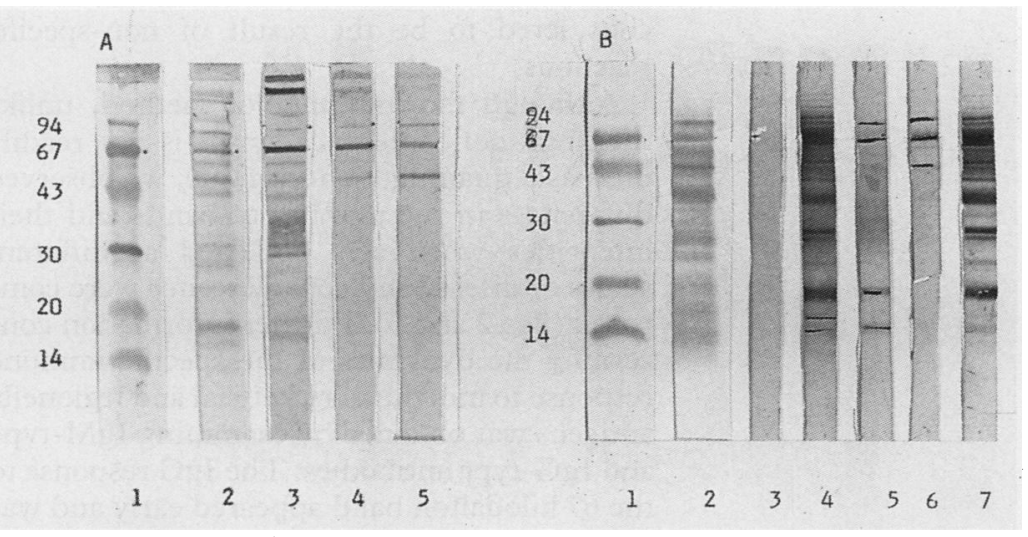

Figure 1 Immunoblot reference profiles of $R$ typhi $(A)$ and $L$ bozemanii $(B)$ antigens with the following rabbit immune sera: anti- (lanes $A 3, B 6)$; anti$L$ bozemanii (lanes $A 5, B 4, B 7$ ); anti-OX19 (lanes $A 4, B 5$ ); control serum from a rabbit before immunisation $(B 3)$. The protein molecular weight standards lanes 1 ( $A$ and $B$ ), and the structural antigens, lanes 2 ( $A$ and $B$ ) were stained with amido-black.

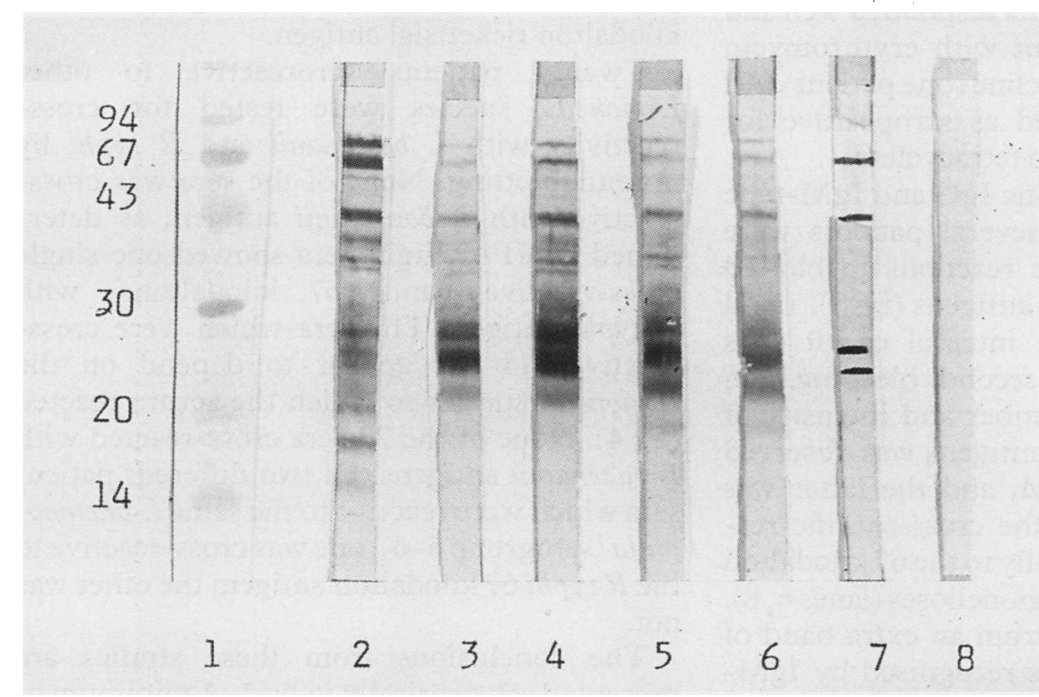

Figure 2 Immunoblot profiles of $L$ bozemanii antigens with two consecutive sera from three patients $(p): p A$ (lanes 3,4$) ; p B$ (lanes 5,6$) ; p C$ (lane 7 ). The protein molecular weight standards are in lane 1 . The reference system is in lane 2. Control sera from bloodbank is in lane 8 .

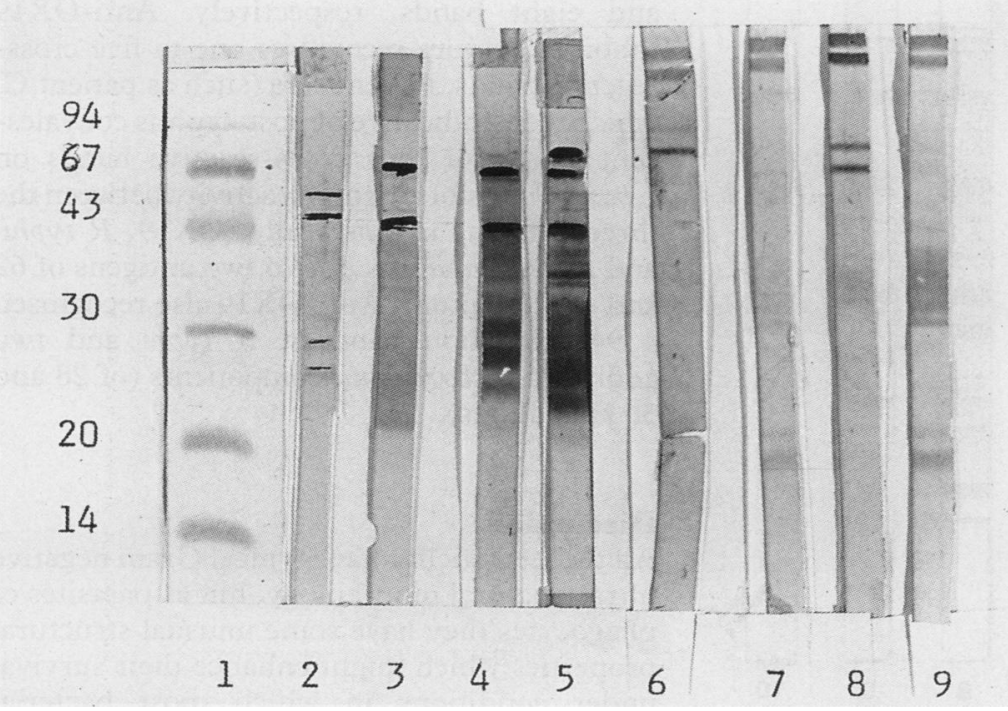

Figure 3 Immunoblot profiles of $L$ bozemanii (lane 2, 3, 4, 5) and $R$ typhi (lane 6, 7, 8,9) antigens with paired patient sera and antihuman Ig G (lane 3, $5,7,9)$ and IgM (lane 2, 4, 6, 8). Serum number 1 is in lane 2, 3, 6, 7. Serum number 2 is in lane 4, 5, 8, 9. The protein molecular weight standards are in lane 1. in culture medium and lysed by one freezethaw cycle and 20 seconds in sonicator waterbath. Rickettsiae were then purified by differential centrifugation ${ }^{10}$ and banded on discontinuous gradients of ditrizoate salts $(25 \%$, $36 \%, 42 \%$; Urografin, Scherring, Germany). Protein concentration of antigen preparations were determined by a modified Lowry technique. ${ }^{11}$ The procedure used to immunise rabbits with these antigens has been described by Harboe and Ingild. ${ }^{12}$

The sonicated antigens were separated by sodium-dodecyl-polyacrylamide gel electrophoresis (SDS-PAGE), as described previously. ${ }^{1314}$ Antigenic fractions on the western blots were visualised (following additional washing) using the chromogenic substrate, 3 amino 9 ethylcarbazole $(0.04 \%$ in $50 \mathrm{mM}$ sodium acetate buffer, $\mathrm{pH} 5.0$, containing $0.018 \% \mathrm{v} / \mathrm{v} \mathrm{H}_{2} \mathrm{O}_{2}$ ).

\section{Results}

The immunoblot profiles of $R$ typhi and $L$ bozemanii structural antigens are shown in fig 1. These homologous and heterologous reactions serve as reference systems for the subsequent study with patient antisera. The homologous reactions fig $1 \mathrm{~A}$, lane 3 (anti- $R$ typhi antiserum with $R$ typhi antigen) and fig $1 \mathrm{~B}$ lanes 4 and 7 (anti- $L$ bozemanii with $L$ bozemanii antigen) are characterised by a multitude of bands, while heterologous responses (fig 1A, lanes 4 and 5, and fig $1 \mathrm{~B}$, lanes 5 and 6 ) show significant humoral responses to three antigens $(94,67$, and 43 kilodaltons) when used to probe $L$ bozemanii blots. Two higher molecular weight bands more than 94 kilodaltons always appeared on $R$ typhi immunoblots, even when probed with normal human serum (including sera from neonates). The reaction against them must therefore be considered non-specific.

Sixty seven sera from 26 patients (between two and four sera per patient) were studied by western blotting. Ten patients were found to seroreact with 12-14 L bozemanii antigen bands and (cross)react with three $R$ typhi antigens. Figure 2 shows the immunoblot profile of $L$ bozemanii antigen recognised by three different patient sera (A, B, and C). The homologous reference system (rabbit anti- $L$ bozemanii) was included for comparison (lane 2). Paired sera from patient A (lanes 3-acute, and 4-convalescent) showed a time dependent increase in the intensity of several bands, probably due to a rise in the antibody titre. The opposite was true for patient $B$ (the intensity of bands in lane 6 is less than that seen in lane 5). These two patients ( $A$ and $B$ ) were diagnosed as having legionellosis, in view of the multitude of bands which were comparable with the homologous reference system. On the other hand, patient $C$ showed only the four bands typified by the heterologous reference system and was thus considered to have had typhus.

These results correlated well with the clinical findings. Eight of these 10 patients showed some degree of respiratory tract disease, which is much more characteristic of legionellosis 


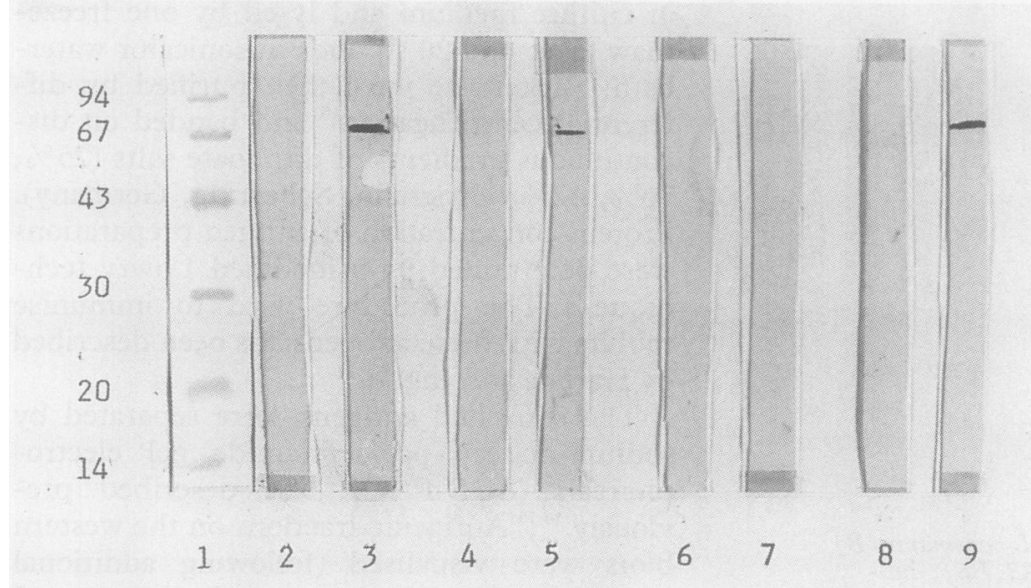

Figure 4 Immunoblot profiles of $L$ bozemanii (lanes 2, 4, 6, 8) and of $R$ typhi (lanes $3,5,7,9$ ) antigens, reacted with seropositive patient sera to

$L$ pneumophila sg 1-4 (lanes 2,3 ); L longbeachae (lanes 4, 5);

$L$ pneumophila sg 5, 6 (lanes 6, 7); L gormanii (lanes 8, 9). The amido black stained protein molecular weight standards are in lane 1.

than of typhus. All patients responded well and recovered after treatment with erythromycin (nine patients) or tetracycline (one patient). All the 16 patients classified as seropositive for typhus responded well to tetracycline.

The dynamics of specific IgG and IgM-type antibody responses in several patients were studied by type specific reactions to blotted $L$ bozemanii and $R$ typhi antigens (fig 3 ). In all cases there was a time interval of 10 days between the first and second bleeding. An increase in the band number and intensity of reaction to $L$ bozemanii antigens was observed with both IgG and IgM, and the latter was mainly responsible for the cross-specific response to $R$ typhi, especially to the 67 kilodalton band seen in definitive legionelloses (lanes 6,8 ). In the second patient serum an extra band of about 50 kilodaltons was recognised by IgMtype antibodies. The two larger bands (more than 95 kilodaltons) in lanes 6-9 (fig 2) were
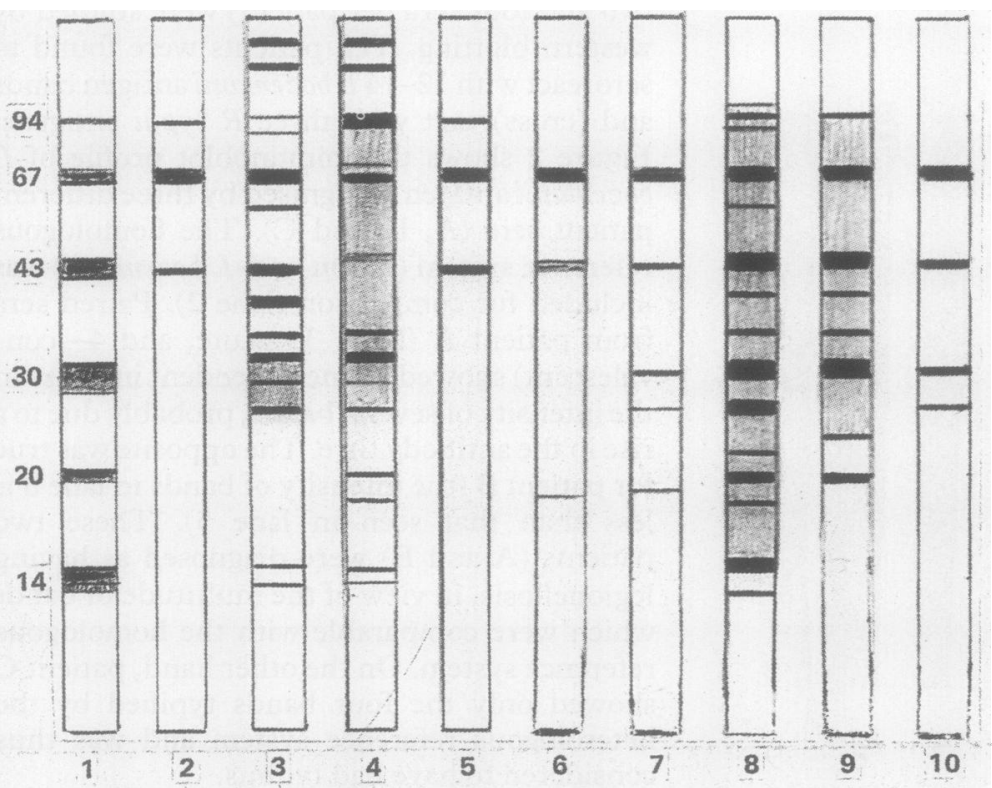

Figure 5 Diagrammatic representation of the banding patterns obtained on $P A G E$ of $R$ typhi (lanes 2-5) and L bozemanii (lanes 6-10) antigens immunoblotted with: rabbit anti-R typhi sera (lanes 3,6 ); anti-L bozemanii sera (lanes 2,8); anti-OX19 sera (lanes 5, 7); "typhus" patient sera (lanes 1, 10); and "legionellosis" patient sera (lane 9). considered to be the result of non-specific reactions.

Although the immunoblot method, unlike the immunofluorescence assay, is not readily used as a quantitative technique, we observed differences in the number of bands and their intensities when sera collected at different stages of disease and convalescence were compared (figs 2 and 3). Further information concerning the dynamics of the specific immune response to individual rickettsial and legionella antigens was obtained by examining IgM-type and IgG-type antibodies. The IgG response to the 67 kilodalton band appeared early and was seen in the acute phase, while the predominant response of $\operatorname{IgM}$ antibodies was to the 43 kilodalton band, with weaker reactivity to 50 and 30 kilodalton legionella antigens and no homologous IgM response to the 67 legionella antigen. Using heterologous ( $R$ typhi) antigen, however, the major early IgM response in patients with legionellosis was against the 67 kilodalton rickettsial antigen.

Twenty patients seroreactive to other Legionella species were tested for crossreactivity with $L$ bozemanii and $R$ typhi by immunoblotting. None of the sera was crossreactive with $L$ bozemanii antigen, as determined by IFA. Eight sera showed one single cross-reactive band (67 kilodaltons) with $R$ typhi antigen. The sera which were crossreactive did not appear to depend on the Legionella species to which the serum reacted (fig 4). None of the 20 sera cross-reacted with $L$ bozemanii antigens. Of two different patient sera which were reactive to the same $L$ pneumophila (serogroup 5-6), one was cross-reactive to the $R$ typhi 67 kilodalton antigen; the other was not.

The conclusions from these studies are presented schematically in fig 5. A minimum of 14 bands was obtained on homologously probed immunoblots of $R$ typhi antigen with either human or rabbit anti-typhus antisera. Homologous $L$ bozemanii immunoblots with human or rabbit anti-L bozemanii sera gave 15 and eight bands, respectively. Anti-OX19 rabbit antiserum recognises one to five crossreactive bands. Patient sera (such as patient $C$ ) which were indicative of post-typhus convalescent sera gave four cross-reactive bands on $L$ bozemanii blots. Cross-reactivity between the three antigens in this study (OX19, $R$ typhi, and $L$ bozemanii was due to two antigens of 62 and 43 kilodaltons. Anti-OX19 also recognised a 94 kilodalton band of $R$ typhi and two additional $L$ bozemanii components (of 28 and 30 kilodaltons).

\section{Discussion}

All the Legionellae have typical Gram negative ultrastructural morphology, but as parasites of phagocytes they have some unusual structural properties which might enhance their survival under conditions in which most bacterial species would die. The peptidoglycan of Legionella is highly cross-linked, and protease resistant proteins have been shown to be in close association with the glycan backbone of 
this structure. ${ }^{7}$ In this respect it resembles the structural relation between the peptidoglycan and other cell wall components of Coxiella burnetii. ${ }^{715}$ The major outer membrane proteins of $L$ pneumophila have been the subject of detailed investigations. They consist of two heat modifiable and immunologically related components of 29 and 45 kilodaltons which bear epitopes common to a wide variety of serotypes. They were found to be strongly associated with lipopolysaccharides. Serogroup specificity is determined by the polysaccharide structure of this component and is recognised by the indirect fluorescence antibody assay. ${ }^{16-18}$ Isolation and further characterisation of these antigens, and using them for consecutive absorption of rabbit and patient antisera, will confirm their role in the immunofluorescence assay. Using a somewhat different approach, murine monoclonal antibodies can be used to map the epitopes responsible for strain, species, and genus specificity in addition to those responsible for crossreactivity of the type described in this study. Such an approach will complement studies evaluating the polyclonal response seen in patients' sera against these pathogenic agents.

1 Wilkinson HW, Fikes BJ, Cruce DD. Indirect immunofluorescence test for serodiagnosis of Legionnaires' disease: evidence for serogroup diversity of Legionnaires' disease bacterial antigens and for multiple specificity of human antibodies. J Clin Microbiol 1979;9:379-83.

2 Thacker WL, Plikaytis BB, Wilkinson HW. Identification of 22 Legionella species and 33 serogroups with the slide agglutination test. J Clin Microbiol 1985;21:779-82.

3 Wilkinson $\mathrm{HW}$, Farshy CE, Fikes BJ, et al. Measure of immunoglobulin $G, M$ and $A$ specific titers against nonspecific, Gram-negative bacterial antigens in the indirect pecific, Gram-negative bacterial antigens in the indirect immunofluorescen

4 Wilkinson HW, Cruce DD, Broome CV. Validation of
Legionella pneumophila by indirect immunofluoroscence assay with epidemic sera. J Clin Microbiol 1981;13: $139-46$

5 Sompolinsky D, Boldur I, Goldwaser RA, et al. Serological cross-reactions between Rickettsia typhi, Proteus vulgaris OX19, and Legionella bozemanii in a series of febrile patients. Isr J Med Sci 1986;22:745-52.

6 Cielsky CA, Blaser MJ, Wang WL. Serogroup specificity of Legionella pneumophila is related to lipopolysaccharide characteristics. Infect Immun 1986;51:397-404.

7 Hindahl MS, Iglewski BH. Outer membrane proteins from Legionella pneumophila serogroups and other Legionella Legionella pneumophila serogroups an

$8 \mathrm{CDC}$. Legionella indirect fluorescent antibody research reagents (revised). Atlanta: Centers for Disease Control, 1985:1-13.

9 Cwikel BJ, Ighbarieh J, Sarov I. Characterisation of antigenic polypeptides from endemic strains of Israel spotted fever (ISF) and comparison with other spotted fever group rickettsia. Rickettsiology, Current Issues and Perspectives. Ann NY Acad Sci 1990;590:381-8.

10 Feng HM, Kirkman C, Walker DH. Radio-immunoprecipitation of (35s) methionine-radiolabeled proteins of Rickettsia conori and Rickettsia rickettsia. J Infect Dis 1986;154: 717-21.

11 Markwell MAK, Haar WM, Biever LL, Tolbert NE. A modification of the Lowry procedure to simplify protein determinations in membrane and lipoprotein samples. Ann Biochem 1978;87:206-10.

12 Harboe N, Igild A. Immunization, isolation of immunoglobulins, estimation of antibody titre. In: Axelson $\mathrm{AH}$ Kroll J, Weeke B, eds. A Manual of Quantitative Immunoelectrophoresis Methods and Applications. Oslo: Universitetsor laget, 1973:1961-4.

13 Hanuka N, Glasner M, Sarov I. Detection of IgG and IgA antibodies to Chlamydia trachomatis in sera of patients with chlamydial infections: use of immunoblotting and immunoperoxidase assays. Sex Transm Dis 1988;15:93-9.

14 Towbin H, Staehelin T, Gordon J. Electrophoretic transfer of proteins from polyacrylamide gels to nitrocellulose of proteins from polyacrylamide gels to nitrocellulose sheets: procedure and some

15 Amano KJ, Williams TF, McCaul FT, Peacock MG. Biochemical and immunological properties of Coxiella burnettii cell wall and peptidoglycan-protein complex fractions. $J$ Bacteriol 1984;150:982-8.

16 Gabay JE, Horwitz MA. Isolation and characterization of the cytoplasmic and outer membranes of the Legionnaires' disease bacterium (Legionella pneumophila). J Exp Med 1985;161:409-22.

17 Boissinot M, Ramsay D, Barthe C, Oly RJ. Antigenic variability of the outer membrane antigens of Legionella pneumophila serogroups 1 to 8. Can J Microbiol 1986; 33:607-13.

18 Nolte FS, Colin CA, Mothley MA. Electrophoretic and serological characterization of the lipopolysaccharide of Legionella pneumophia. Infect Immun 1986;52:676-81. 